\title{
Enhanced cyanine solar cell performance upon oxygen doping
}

\author{
Bin Fan ${ }^{\text {a }}$, Roland Hany ${ }^{\text {a }}$, Jacques-Edouard Moser ${ }^{\mathrm{b}}$, Frank Nüesch ${ }^{\mathrm{a}, *}$ \\ a Laboratory for Functional Polymers, Swiss Federal Laboratories for Materials Testing and Research, Empa, \\ Überlandstrasse 129, CH-8600 Dübendorf, Switzerland \\ b Photochemical Dynamics Group, Institute of Chemical Sciences and Engineering, Ecole Polytechnique Fédérale de Lausanne, \\ CH-1015 Lausanne, Switzerland
}

Received 29 July 2007; received in revised form 25 September 2007; accepted 30 September 2007

Available online 13 October 2007

\begin{abstract}
The effect of exposing cyanine-fullerene $\mathrm{C}_{60}$ bilayer solar cells to ambient atmosphere is investigated. For exposure times of a few hours and concomitant light soaking, the device performance experiences a drastic power efficiency increase going from $0.14 \%$ to $1.2 \%$ measured at $30 \mathrm{~mW} / \mathrm{cm}^{2}$ simulated solar irradiation. The 10 -fold enhancement is attributed to the photoinduced doping involving oxygen and water leading to the formation of reactive superoxide anions and mobile holes in the cyanine layer. The influence of water and dry oxygen are investigated separately. While water deteriorates the device performance, dry oxygen leads only to a partial increase of efficiency. Annealing does not ameliorate the performance of doped devices. Although then the cyanine layer features more crystallinity, the considerable morphological changes cause diffusional loss in charge carrier collection. Doping of not annealed devices brings a sizeable efficiency enhancement that highlights the importance of charge carrier transport in cyanine dye based solar cells.
\end{abstract}

(C) 2007 Elsevier B.V. All rights reserved.

PACS: $72.80 . \mathrm{Jc} ;$ 84.60.Jt; 78.40.Me; 73.61.Ph; 27.40.tw; 78.30.Jw; 73.50.Pz

Keywords: Organic optoelectronic devices; Photovoltaic cells; Solar cells; Cyanine dyes; Fullerene; $\mathrm{C}_{60}$; Doping; Oxygen; Annealing; Charge transport; Superoxide anion; Conductivity

\section{Introduction}

Cyanine dyes were developed at the beginning of the 20th century, mainly as sensitizers for silver halide emulsions in the photographic process [1]. Above all, cyanines exhibit extraordinarily high extinction coefficients and tunable absorption spectra throughout the visible and near infrared domain.

\footnotetext{
${ }^{*}$ Corresponding author. Tel.: +41 448234740.

E-mail address: frank.nueesch@empa.ch (F. Nüesch).
}

Because of their unique optical properties, they have more recently been applied in non-linear optics [24], in data storage devices [5], as fluorescent probes in biomolecules [6] or as contrast agents in optical imaging of tissue [7]. Redox properties of cyanine dyes have also been extensively studied with respect to their role as sensitizer or desensitizer for silver halides [8]. The wide range of oxidation and reduction potentials allows cyanines to act as electron donors as well as electron acceptors in photoinduced electron transfer processes. 
Combined advantages of optical and electronic properties make cyanines an interesting material class for solar cell applications. Numerous works report on cyanines used in dye sensitized solar cells [9-14]. Power efficiencies greater than $5 \%$ and current densities exceeding $21 \mathrm{~mA} / \mathrm{cm}^{2}$ at AM $1.5 \mathrm{sim}$ ulated solar irradiation have been found. Despite these promising results, photoinduced degradation of related polymethine dyes due to titanium dioxide catalysed decomposition reactions has been reported $[15,16]$ and might also be a concern for cyanines. This degradation pathway can be avoided in solid all-organic solar cell devices, which also allow cost efficient fabrication processes. Only few works report on organic solar cells using solid cyanine films as active material [17-19]. So far, these devices have shown rather modest efficiencies which could be related to inefficient charge transport.

Different approaches to increase charge transport in organic solar cells have been proposed. Crystallinity can be induced by annealing and often leads to enhanced charge transport [20-23]. Another way to improve charge transport relies on enhancing the conductivity of the organic film. Based on the pioneering work on semiconducting and metallic polymers [24], electrical conductivity has been induced by doping various optoelectronic materials with oxidizing or reducing agents [25-27]. This strategy has been used to fabricate organic $\mathrm{p}-\mathrm{i}-\mathrm{n}$ junction [28] and Schottky barrier solar cells [29]. In some cases enhancing the bulk film conductivity in organic photovoltaic cells proved to be beneficial for device efficiency [30,31].

In this work we investigate the effect of exposing cyanine dye- $\mathrm{C}_{60}$ bilayer devices to ambient atmosphere under light irradiation. The drastic increase of performance upon short exposure to air is explored by separately looking at the influence of water and oxygen. The impact of light irradiation during air exposure on the device characteristics is analysed in order to get insight into the reaction mechanism. Finally the effect of annealing is discussed.

\section{Experimental}

\subsection{Materials}

Cyanine dye 1,1'-diethyl-3,3,3',3'-tetramethylcarbocyanine perchlorate $(\mathrm{Cy}-5)$ was synthesized in our laboratory and recrystallized in ethanol before use. Poly(3,4-ethylenedioxythiophene)-poly(styrenesulfonate) (PEDOT:PSS, Bayer), fullerene $\mathrm{C}_{60}$ (SES
Research, 99.95\% purity) and tetrafluoropropanol (TFP, Fluka) were used as received. The chemical structures of the organic compounds used in this work are shown in Fig. 1.

\subsection{Cyclic voltammetry}

Cyclic voltammetry measurements were recorded on a PGStat 30 potentiostat (Autolab) using a three cell electrode system with a glassy carbon working electrode, a platinum counter electrode and an $\mathrm{Ag} / \mathrm{AgCl}$ (0.1 M tetrabutyl ammonium chloride in acetonitrile) reference electrode. Redox potentials of Cy-5 were measured in acetonitrile and referenced to the ferrocene/ferrocenium couple. To relate the electrochemical potentials to the corresponding vacuum levels, the potential of $0.69 \mathrm{~V}$ vs NHE was taken for ferrocene and the NHE value of $-4.48 \mathrm{eV}$ vs vacuum was adopted from the literature [32]. Redox potentials of $\mathrm{C}_{60}$ referenced to ferrocene/ferrocenium were taken from the literature [33]. Therefore the same relationship between the electrochemical scale and the physical scale was applied to obtain the vacuum reduction and oxidation potentials. The highest occupied molecular orbital energy level of PEDOT:PSS was adopted from the literature [34]. Fig. 1 summarizes lowest unoccupied molecular orbital (LUMO) and highest occupied molecular orbital (HOMO) energy levels of the different materials used in this work.

\subsection{Device fabrication and characterization}

Solar cell devices were fabricated on patterned indium-tin-oxide coated glasses (ITO, $140 \mathrm{~nm}$, $20 \Omega$ /square) that were cleaned in ethanol, acetone and detergent ultrasonic baths. A $90 \mathrm{~nm}$ thick PEDOT:PSS layer was deposited by spin-coating and heated in vacuum at $150{ }^{\circ} \mathrm{C}$ for $10 \mathrm{~min}$ to get rid of residual water. A $40 \mathrm{~nm}$ thick $\mathrm{Cy}-5$ layer was then spin-coated from a $5 \mathrm{mg} / \mathrm{ml}$ solution in TFP on top of PEDOT:PSS, followed by vapour depositon of a $40 \mathrm{~nm}$ thick $\mathrm{C}_{60}$ layer under high vacuum. Finally a $70 \mathrm{~nm}$ thick aluminium cathode was vapour deposited through a shadow mask, defining four separate solar cell devices with an active device area of $0.031 \mathrm{~cm}^{2}$. The full device fabrication sequence leading to the ITO/PEDOT:PSS/Cy-5/ $\mathrm{C}_{60} / \mathrm{Al}$ architecture was carried out under inert atmosphere. To study doping effects, devices were exposed to ambient atmosphere for several hours in the dark or under room light irradiation of $5 \mathrm{~mW} \mathrm{~cm}^{-2}$. In 


\section{Cy5}

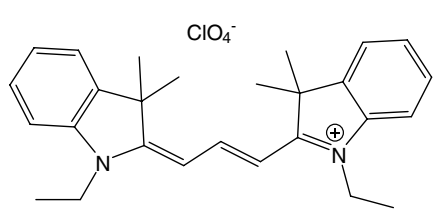

PEDOT:PSS

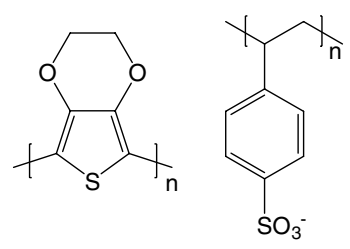

$\mathrm{C}_{60}$

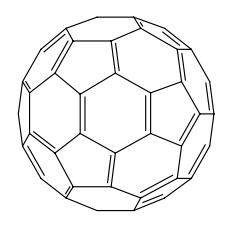

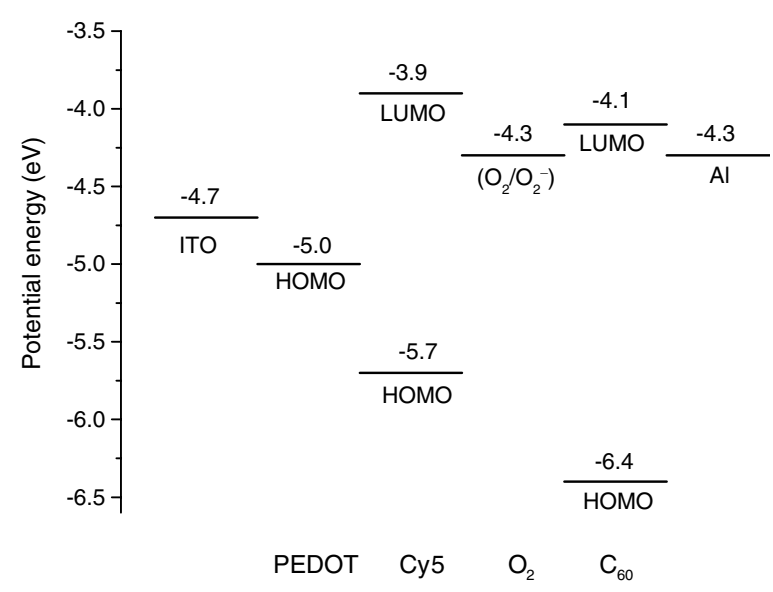

Fig. 1. Chemical structures of the materials used in this work. The HOMO and LUMO energy levels, the workfunctions of the electrodes as well as the reduction potential of triplet oxygen are also indicated.

order to investigate the effect of water separately, samples were placed in a glass decicator under the same irradiation conditions. A special measurement cell was constructed to characterize devices that were subjected to a flow of water saturated nitrogen. Doping was also studied for annealed devices. In this case the completed devices were heated in a vacuum oven at $100{ }^{\circ} \mathrm{C}$ for various durations.

Thicknesses of the organic films were measured using an Ambios XP-1 profilometer, while morphology analyses were carried out on an Nanosurf Mobile $\mathrm{S}$ atomic force microscope in tapping mode employing rectangular silicon cantilevers (Mikromasch, Nanosensors). Optical absorption spectra were measured with a Varian Cary 50 spectrophotometer.

Photovoltaic device performance was characterized under nitrogen atmosphere or in air using a monochromator (Oriel 74000) with a xenon lamp light source to obtain the incident photon-to-current conversion efficiency (IPCE) according to $\mathrm{IPCE}=1240 \times J_{\mathrm{SC}} \times \lambda^{-1} \times P_{\text {in }}^{-1}$ where $J_{\mathrm{SC}}(\mathrm{mA} /$ $\left.\mathrm{cm}^{2}\right)$ is the short-circuit current-density, $P_{\text {in }}(\mathrm{mW}$ $\mathrm{cm}^{-2}$ ) the incident light intensity at wavelength $\lambda$ $(\mathrm{nm})$. Current-voltage characteristics were recorded using a Keithley 2400 source-measure unit. Simulated solar spectra with irradiation intensities of $30 \mathrm{~mW} / \mathrm{cm}^{2}$ or $47 \mathrm{~mW} / \mathrm{cm}^{2}$ were obtained from the same xenon discharge lamp using an AM1.5G filter set (Oriel). Power efficiencies were calculated as $\eta=\mathrm{FF} \times J_{\mathrm{SC}} \times V_{\mathrm{OC}} \times P_{\text {in }}^{-1}$, where $\mathrm{FF}=V_{\mathrm{m}} \times$ $J_{\mathrm{m}} \times V_{\mathrm{OC}}^{-1} \times J_{\mathrm{SC}}^{-1}$ is the fill factor, $V_{\mathrm{m}}(\mathrm{V})$ and $J_{\mathrm{m}}$ $\left(\mathrm{mA} / \mathrm{cm}^{2}\right)$ are the current-density and voltage at maximum power output, $V_{\mathrm{OC}}(\mathrm{V})$ is the open-circuit voltage.

\subsection{Four probe measurements}

Electrical conductivity measurements of thin Cy5 films were performed by a standard four-probe method. The glass substrates comprised four finger-like gold electrodes separated by a channel distance of $10 \mu \mathrm{m}$. A current of $10 \mathrm{nA}$ was applied at the outer electrodes while the voltage was measured at the inner electrodes.

\section{Results and discussion}

In order to investigate the effect of exposing ITO/PEDOT:PSS/Cy-5/C $60 / \mathrm{Al}$ devices to ambient 
atmosphere, solar cells were first characterized in nitrogen atmosphere without being exposed to the ambient atmosphere at any time. Since the device fabrication procedure excludes any contact with air, it can be assumed that freshly prepared devices only contain low amounts of residual water and oxygen. Then they were exposed to air for several hours and characterized in regular time intervals. Fig. 2 shows the effect of air soaking on device performance, by indicating the variation of short-circuit current and open-circuit voltage. A steep rise in $V_{\text {OC }}$ can be observed within the first two hours leading to an increase from $0.47 \mathrm{~V}$ to $0.60 \mathrm{~V}$. At longer exposure times $V_{\mathrm{OC}}$ gradually saturates at $0.73 \mathrm{~V}$. With some retardation, the short-circuit current follows the rise of the open-circuit voltage, reaches a maximum at $1.8 \mathrm{~mA} / \mathrm{cm}^{2}$ and starts to drop. At exposure times of a few days, the device characteristics severely degrade and ultimately drop-off below the initial value.

The maximum power efficiency is reached for devices that were soaked in air and light for a duration of $3 \mathrm{~h}$. The IPCE doubles with respect to the initial value (Fig. 3a) and the current increases in a spectacular way leading to a power efficiency of $1.2 \%$. As can be inferred from Table 1 the fill factor also rises from 0.19 to 0.27 , indicating increased charge carrier collection.

Different mechanisms may be invoked to explain the rise in device performance. Oxygen could assist charge generation in the cyanine layer and augment photocurrent generation in the bulk. This hypothesis can be discarded based on the Cy-5 thickness dependence of the external quantum efficiency. At

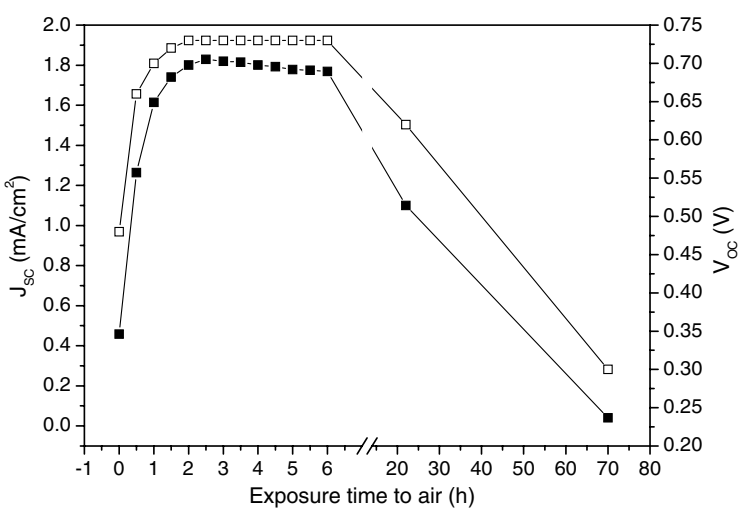

Fig. 2. Variation of the short-circuit current $J_{\mathrm{SC}}$ (full squares) and the open-circuit voltage $V_{\mathrm{OC}}$ (empty squares) of ITO/ PEDOT:PSS/Cy-5/C $60 / \mathrm{Al}$ devices as a function of exposure time to ambient atmosphere and white light irradiation.
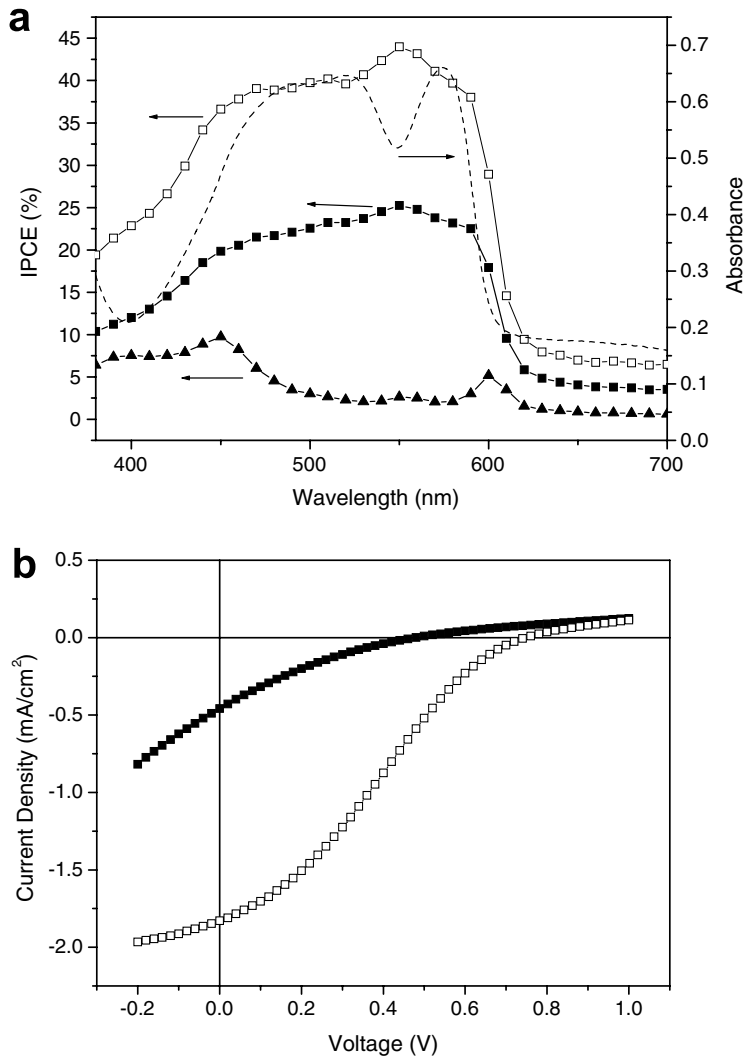

Fig. 3. ICPE and absorbance (a) and current-voltage characteristics (b) of ITO/PEDOT:PSS/Cy-5 $(40 \mathrm{~nm}) / \mathrm{C}_{60}(40 \mathrm{~nm}) / \mathrm{Al}$ devices right after fabrication (full squares) and after being exposed to ambient atmosphere and white light irradiation for $3 \mathrm{~h}$ (empty squares). Additionally a device using a $100 \mathrm{~nm}$ thick Cy-5 layer that was also exposed to air is shown in (a) (full triangles). Devices were measured at an irradiation intensity of $30 \mathrm{~mW} \mathrm{~cm}^{-2}$.

Table 1

Photovoltaic characteristics of ITO/PEDOT:PSS/Cy-5/C $60 / \mathrm{Al}$ devices measured just after fabrication (fresh) and soaked in ambient atmosphere under white light irradiation of $5 \mathrm{~mW} \mathrm{~cm} \mathrm{~cm}^{-2}$ for the duration of $3 \mathrm{~h}$ (air)

\begin{tabular}{lllll}
\hline & $V_{\mathrm{OC}}(\mathrm{V})$ & $J_{\mathrm{SC}}\left(\mathrm{mA} / \mathrm{cm}^{2}\right)$ & $\mathrm{FF}$ & $\eta(\%)$ \\
\hline Fresh & 0.47 & 0.46 & 0.189 & 0.14 \\
Air & 0.73 & 1.83 & 0.274 & 1.2 \\
\hline
\end{tabular}

The devices were measured at a white light irradiation intensity of $30 \mathrm{~mW} \mathrm{~cm}^{-2}$.

thicknesses above $50 \mathrm{~nm}$, the IPCE does not follow the absorption spectrum but shows a marked filter effect with a minimum in the spectral region where Cy-5 absorbs strongly and two maxima at the absorption edges of the cyanine (see Fig. 3a). This behaviour is similar to non-exposed devices that were reported in a previous work [19]. At a thick- 
ness of $40 \mathrm{~nm}$ the filter effect is still apparent in the IPCE maximum corresponding to the local minimum in the absorption spectrum at $550 \mathrm{~nm}$ (see Fig. 3a).

Doping by oxygen is therefore proposed as an explanation for the tenfold increase in power efficiency upon air exposure. By looking at the current-voltage curve measured in the dark, a marked increase in the forward current is observed upon air soaking while the reverse current lowers significantly (Fig. 4). This results in a rectification ratio of 151 measured at $\pm 1.5 \mathrm{~V}$, which is a fundamental change as compared to the almost symmetrical current-voltage curve obtained for freshly prepared devices. According to the energy level diagram in Fig. 1, electrons can easily be injected from the $\mathrm{Al}$ cathode under forward bias and are blocked at the $\mathrm{C}_{60}$-cyanine heterointerface, while holes would face a significant injection barrier at the PEDOT:PSS electrode. Therefore the current onset is mainly determined by the injection of holes at the anode. Under reverse bias, holes can hardly be injected from the $\mathrm{Al}$ cathode into $\mathrm{C}_{60}$, and electrons face a considerable injection barrier at the interface between PEDOT:PSS and Cy-5. Similarly to the forward bias situation, injection from the PEDOT:PSS electrode again determines the currentonset under reverse bias.

Rectification of the current-voltage characteristics upon doping with oxygen has indeed been observed in the literature and has been attributed to Schottky barrier formation at the metal electrode [35]. In the present work, p-type doping induced by oxygen would give rise to a Schottky barrier at the anode, favouring hole injection and inhibiting electron injection (Fig. 4a). Note that $\mathrm{Cy}-5$ is a cationic dye with a rather mobile perchlorate counter-anion that can significantly augment charge carrier injection under forward bias due to ion accumulation at the anode. By using fast current-voltage scans, ion motion can be significantly reduced. The reverse current, however, is only slightly affected by ion motion and is therefore a better probe for the formation of a Schottky barrier.

The question still remains regarding the doping mechanism in Cy-5 based devices. To scrutinize the reaction of the cyanine layer in ambient atmosphere, the devices were submitted to air in the dark for $0.5 \mathrm{~h}$. Measured in the dark, the current-density under reverse bias is not reduced to the same amount as under illumination (Fig. 5). Moreover the current is lowered both under forward and reverse bias, if exposed to air for longer durations. As will be shown below, the decreased current-density can be attributed to the effect of water only. Therefore the mechanism of doping leading to rectified current-voltage characteristics must be photoinduced.

In a next step, devices were exposed to dry oxygen under white light irradiation to analyse the effect of oxygen only. In this case, the current-voltage characteristics in the dark show an intermediate behaviour (Fig. 6a). The rectification ratio rises from 1 to 30 after being placed in the decicator and irradiated with $5 \mathrm{~mW} / \mathrm{cm}^{2}$ light for $3 \mathrm{~h}$. Solar cell performance increases substantially but never reaches the performances achieved by the simultaneous action of oxygen and water (Fig. 6b). It
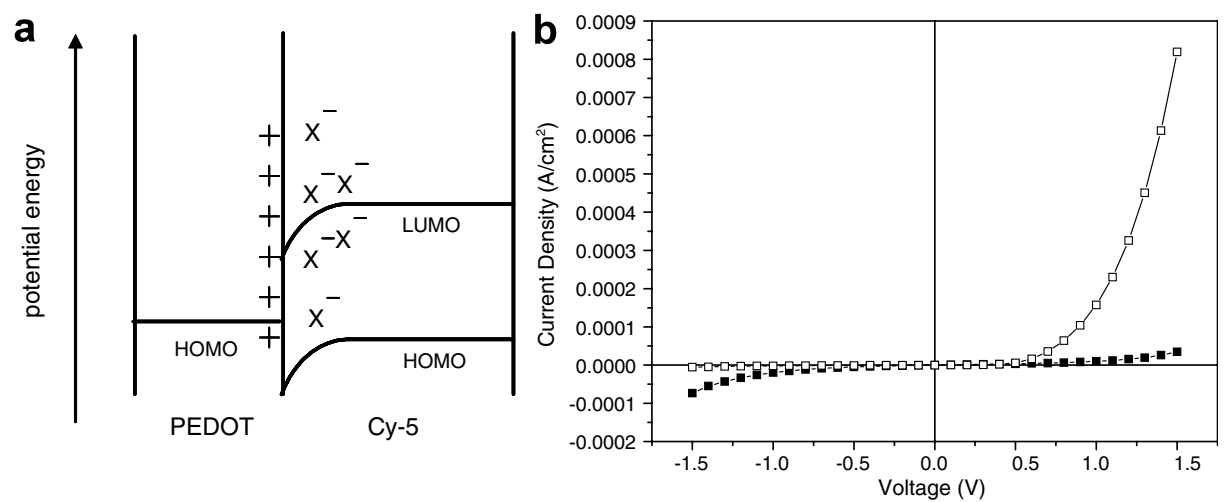

Fig. 4. Schematic drawing of Schottky barrier formation at the PEDOT:PSS interface induced by p-type doping with oxygen (a). The anionic species corresponding to the photochemically created oxygenated complex are labelled as $\mathrm{X}^{-}$, positive charge carriers are marked as plus signs. Figure (b) shows the dark current for a freshly prepared cell (full squares) and a cell that was exposed to ambient atmosphere and white light (empty squares) for $3 \mathrm{~h}$. 


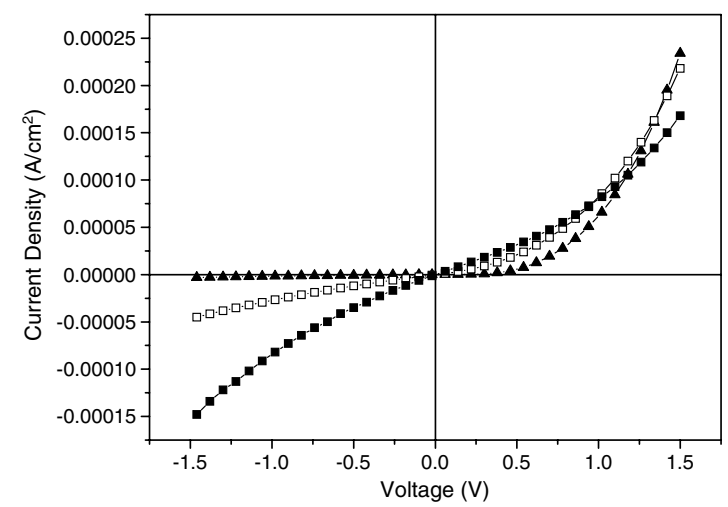

Fig. 5. Current-voltage characteristics of ITO/PEDOT:PSS/Cy$5 / \mathrm{C}_{60} / \mathrm{Al}$ devices measured in the dark just after being fabricated (full squares), after being exposed to ambient atmosphere for $0.5 \mathrm{~h}$ in the absence of light (open squares) and under white light irradiation (full triangles).

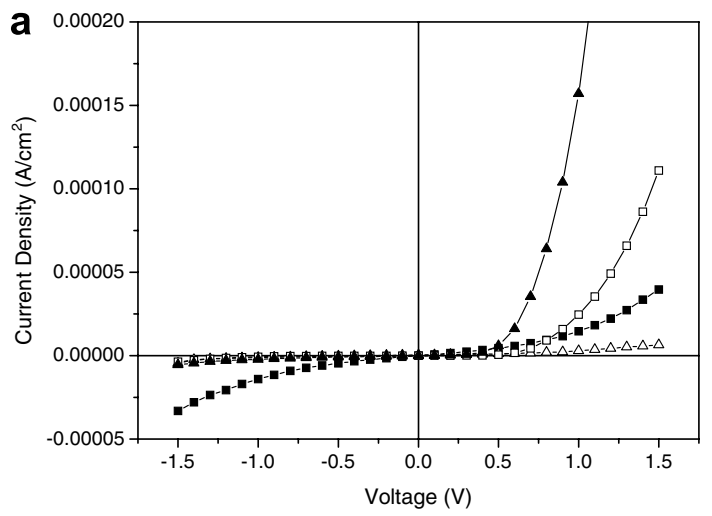

b

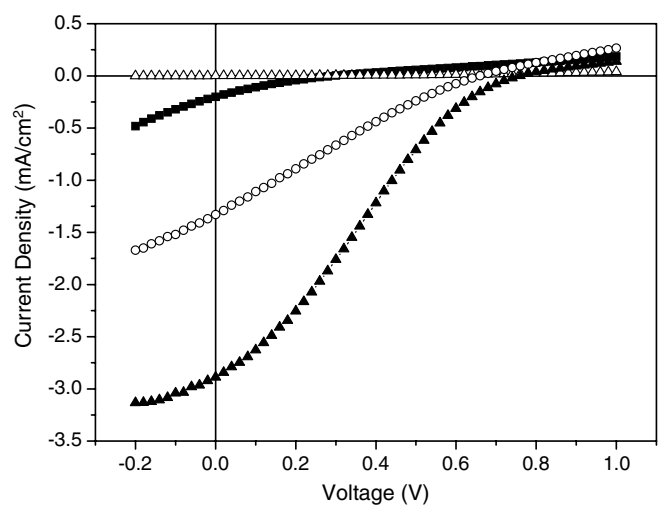

Fig. 6. Current-voltage characteristics of ITO/PEDOT:PSS/Cy$5 / \mathrm{C}_{60} / \mathrm{Al}$ devices just after being fabricated (full squares), after being exposed under white light irradiation to dry air for $3 \mathrm{~h}$ (empty squares), to ambient atmosphere for $3 \mathrm{~h}$ (full triangle) as well as to water saturated nitrogen for $1 \mathrm{~h}$ (empty triangles). (a) Dark current. (b) Devices measured at a simulated solar irradiation intensity of $47 \mathrm{~mW} \mathrm{~cm} \mathrm{~cm}^{-2}$. has to be noted that some water contamination occurred during the transfer of the devices from the glove box to the decicator which may already favour the doping process. Nevertheless, light irradiation occurred under dry atmosphere. The experimental findings therefore emphasize the beneficial effect of water in the doping process.

Water alone, however, has a detrimental effect on all device characteristics. When the cell is exposed to water saturated nitrogen, the current-voltage characteristics measured in the dark gradually decreases both under forward and reverse bias to reach roughly a 10 times smaller current after being immersed in the flow cell for $1 \mathrm{~h}$. The photovoltaic effect reached the noise level of our measurement unit and could no longer be detected (see Fig. 6b). It appears that water can not be the only factor leading to doping. As was shown in the literature, water is absorbed by the hygroscopic PEDOT:PSS layer, thereby decreasing the conductivity of the latter and introducing a large serial resistance to the device [36]. The same phenomenon is likely to occur in the devices described here.

With a view to further improve device performance, fresh ITO/PEDOT:PSS/Cy-5/ $\mathrm{C}_{60} / \mathrm{Al}$ devices were annealed in a vacuum oven at $100{ }^{\circ} \mathrm{C}$ for $30 \mathrm{~min}$. Indeed, a clear rise in the $J_{\mathrm{SC}}$ is observed (Fig. 7a). It can be attributed to a higher mobility due to increased crystallinity of the cyanine film leading to higher charge carrier mobility. Surprisingly, the open-circuit voltage drops by $100 \mathrm{mV}$ and the fill factor decreases from 0.19 to 0.17 , which is contrary to what can be expected from increased charge carrier mobility. In order to find an explanation for this behaviour the morphologies of the devices were analyzed by atomic force microscopy using tapping mode. To avoid peeling-off the cathode thereby possibly destroying the top surface layer, we analyzed the surface area of the organic multilayer that was not covered by aluminium. Non-annealed devices show a fine, granulated structure that homogeneously covers the entire surface (Fig. 7c). In analogy to other bilayer devices using a thin fullerene acceptor layer, this structure is attributed to small $\mathrm{C}_{60}$ spheres on top of the cyanine layer [37]. After annealing, the sphere like features are no longer homogeneously distributed over the surface. Instead, larger rectangular shapes appear additionally to the small spheres, and in some cases the former protrude to the top surface. These larger crystallites are attributed to cyanine aggregates. Similar morphological changes may occur in the film 

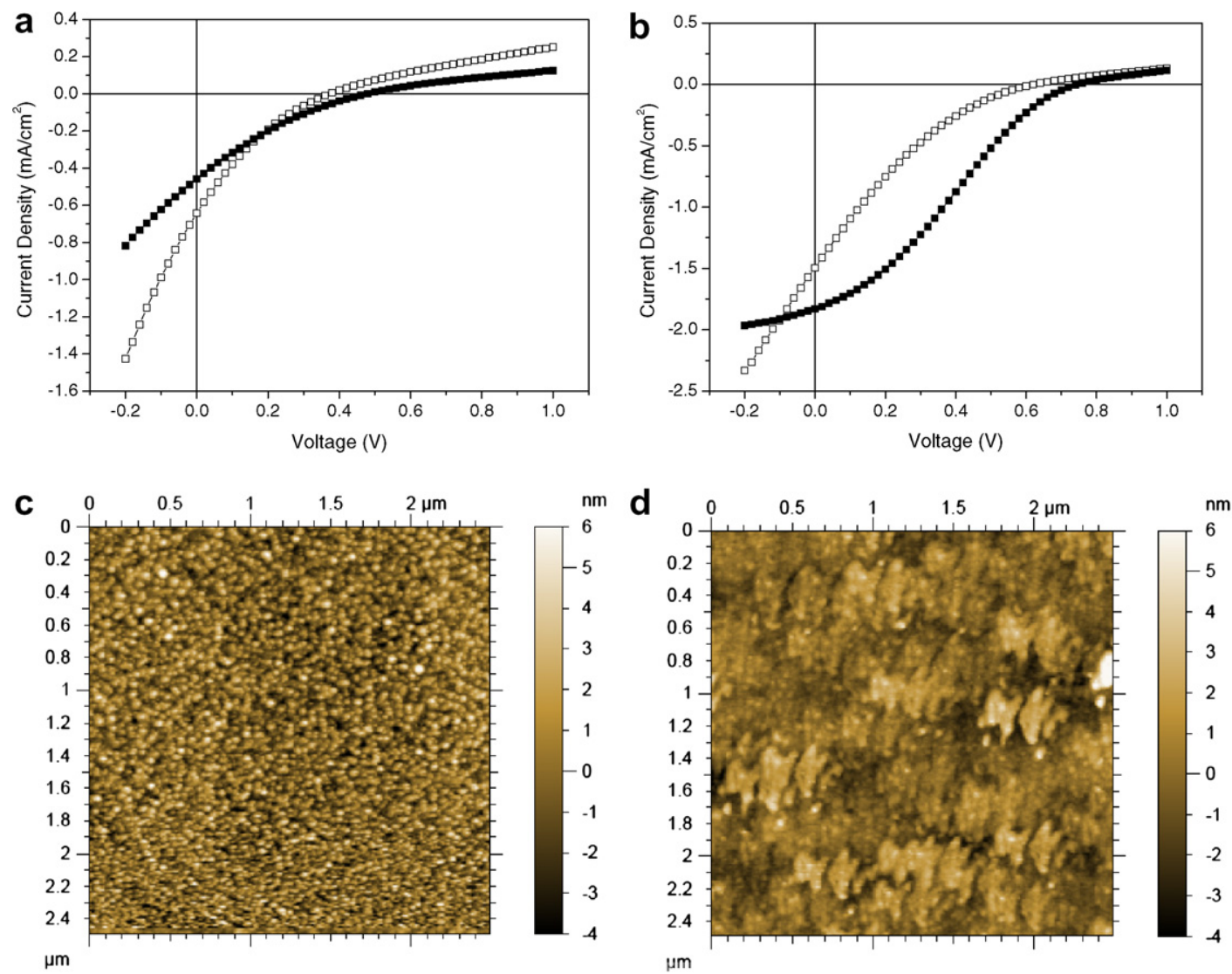

Fig. 7. Current-voltage characteristics of ITO/PEDOT:PSS/Cy-5/ $\mathrm{C}_{60} / \mathrm{Al}$ devices with (empty squares) and without (full squares) being annealed in a vacuum oven at $100{ }^{\circ} \mathrm{C}$ for $30 \mathrm{~min}$. Undoped (a) and doped (b) devices were measured at a simulated solar irradiation intensity of $30 \mathrm{~mW} \mathrm{~cm}^{-2}$. Atomic force microscopy images show the morphology of above doped devices measured on top of the organic layers that were not covered by aluminium for non-annealed (c) and annealed (d) devices.

areas covered by aluminium, giving rise to cyanine and $\mathrm{C}_{60}$ channels that are in direct contact with both anode and cathode. Such a film morphology would allow charge carrier diffusion to both electrodes, which becomes particularly unfavourable for charge carrier collection in the case of low drift fields, i.e. when the applied voltage approaches the open-circuit voltage. As a consequence, lowered open-circuit voltage and fill factor are expected, which may explain the behaviour of annealed devices.

For doped devices the fill factor also decreases upon annealing, from 0.27 to 0.16 for not-annealed and annealed devices, respectively (Fig. 7b). Differently to undoped devices, the short-circuit current is lower for annealed cells as compared to nonannealed ones. However, $J_{\mathrm{SC}}$ can be increased after placing the devices under vacuum for $12 \mathrm{~h}$. As can be inferred from Fig. 8, the short-circuit current rises up to a maximum and then decreases rapidly.

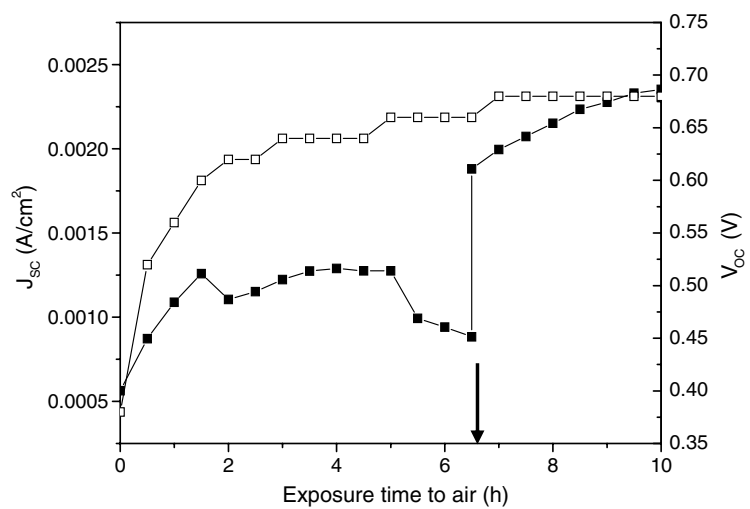

Fig. 8. Variation of short-circuit current $J_{\mathrm{SC}}$ (full squares) and open-circuit voltage $V_{\text {OC }}$ (empty squares) of an annealed ITO/ PEDOT:PSS/Cy-5/ $\mathrm{C}_{60} / \mathrm{Al}$ device as a function of exposure time to ambient atmosphere and white light irradiation. The device was annealed in vacuum at $100{ }^{\circ} \mathrm{C}$ for 30 min before being soaked in air. The vertical arrow indicates the time at which the device was pumped in a vacuum chamber for $12 \mathrm{~h}$. 
This decrease is attributed to the uptake of water by the hygroscopic PEDOT:PSS layer leading to a higher series resistivity. The inhomogeneous, crystalline film morphology opens larger channels in the thin film where water can penetrate more easily. Pumping desorbs water from PEDOT:PSS and restores the short-circuit current.

Above experimental findings demonstrate that photoinduced doping occurs, when devices are soaked in ambient atmosphere for several hours. So far, the precise reaction mechanism has not been addressed. Formation of a rectifying Schottky barrier strongly indicates that doping occurs in the cyanine layer. Moreover, the higher fill factor suggests that hole transport in the cyanine layer is increased. The possibility that the reaction of air with $\mathrm{C}_{60}$ under illumination is responsible for the observed efficiency rise can also be discarded since it has recently been shown that this reaction leads to a decrease of device performance [38]. Photochemical reactions of cyanine dyes with oxygen have indeed been reported in the literature, both in solution and in thin solid films. Self-sensitized energy transfer from the triplet excited state of a cyanine dye to triplet oxygen $\mathrm{O}_{2}\left({ }^{3} \Sigma_{\mathrm{g}}^{-}\right)$leading to reactive singlet oxygen $\mathrm{O}_{2}\left({ }^{1} \Delta_{\mathrm{g}}\right)$ was found to be responsible for photofading of the dye in solution. Evidence was brought indirectly by using a spin trap [39] or by detecting the ketonic reaction products using NMR [40]. Self-sensitized photobleaching due to triplet-triplet energy transfer has also been observed in thin Cy-5 films by monitoring the singlet oxygen emission in the near infrared [41].

Photochemical reaction involving the energy transfer mechanism, however, can not account for the doping process, i.e. the generation of free charge carriers. Electron transfer from the photoexcited state of the cyanine dye to triplet oxygen is also energetically possible given that the redox potential for the reduction of triplet oxygen is at $-0.16 \mathrm{eV}$ vs NHE [42] as compared to the reduction potential of $-0.59 \mathrm{eV}$ vs NHE for Cy-5. The photoinduced electron transfer process has indeed been observed for a cyanine dye in solution by using spin trapping to detect transient superoxide anion $\mathrm{O}_{2}^{-}$species [43]. In one of the works both the electron transfer process and the energy transfer process from photoexcited cyanine to oxygen were observed [39]. Active oxygen species have also been generated from photoexcited fullerene $\mathrm{C}_{60}$ [44]. Interestingly, the authors observed efficient generation of singlet oxygen $\mathrm{O}_{2}\left({ }^{1} \Delta_{\mathrm{g}}\right)$ in nonpolar solvents such as benzene and benzonitrile, while $\mathrm{O}_{2}^{-}$and subsequent formation of hydroxyl radicals were detected instead in polar solvents such as water. Based on above discussion, both energy and electron transfer reaction mechanisms may be present in the photooxygeneation of Cy-5 solid films. If water is coadsorbed together with oxygen, the electron transfer path is favored leading to increased production of positive charge carriers and superoxide anions that further react to yield anionic cyanine oxidation products.

To verify that free charge carriers are indeed generated during the photodoping process, the conductivity of Cy-5 films was directly measured by four probe technique. Cyanine films were drop-cast on glass substrates patterned with four finger-like gold electrodes separated by a channel length of 10 microns. Fig. 9 presents the conductivity change after exposure to ambient atmosphere in the dark as well as under $5 \mathrm{~mW} / \mathrm{cm}^{2}$ white light irradiation. In both cases the conductivity was measured in absence of light. Very clearly there is a strong increase of the conductivity for the irradiated films, while the films kept in the dark show a relatively small variation. This corroborates our hypothesis that photoinduced doping of the cyanine layer occurs during exposure to ambient atmosphere and simultaneous white light irradiation. The conductivity rise in the four probe experiment is much faster than the performance rise observed in solar cells which can be attributed to the oxygen and water barrier effect of the aluminum cathode of the devices. After an exposure time to ambient atmosphere and light of $10 \mathrm{~min}$, the conductivity of Cy-5 films starts to decrease. We have assigned

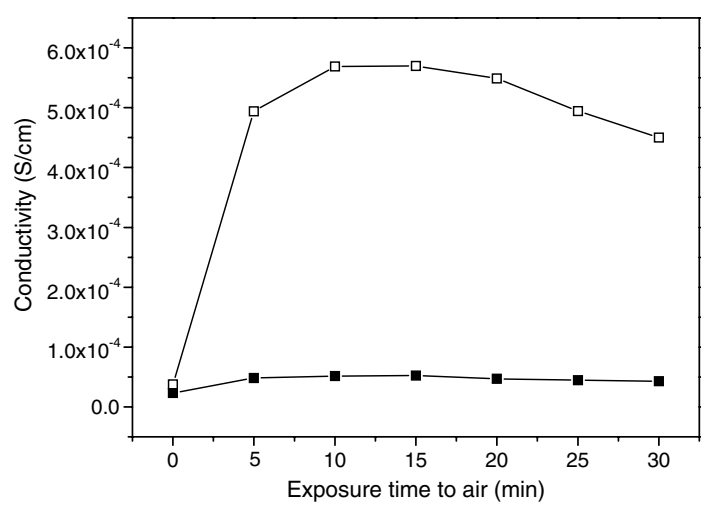

Fig. 9. Conductivity measurements of $\mathrm{Cy}-5$ films as a function of exposure time to ambient atmosphere in the dark (full squares) and under white light irradiation (empty squares). In both cases, conductivity measurements were performed in the absence of light. 
this effect to the degradation of the cyanine film in the vicinity of the gold electrodes. Bubble formation could be observed which may come from hydrogen gas evolution following electrochemical reduction of water during the conductivity measurement.

\section{Conclusions}

We have demonstrated a tenfold increase in power efficiency of cyanine based heterojunction solar cells by doping the cyanine film in the presence of oxygen, water and light. This raises the efficiency of cyanine based thin film solar cells above the $1 \%$ benchmark. The encouraging results highlight the potential of cyanine dyes and the importance to increase charge carrier transport in thin cyanine films. Charge carrier doping is a possible way to achieve high performance. Although promising, the air doping method presents some drawbacks. While water adsorption in PEDOT:PSS leads to increased serial resistivity, photochemical reaction with oxygen can also yield ketonic species acting as charge trapping defects and lowering device efficiency. Well defined chemical doping employing carefully chosen oxidizing agents could therefore even further improve cyanine based devices.

\section{Acknowledgements}

We acknowledge financial support of this work by the Swiss Competence Center for Energy and Mobility CCEM-CH as well as "swisselectric research". Matthias Nagel is thanked for his help in cyclic voltammetry measurements. Thomas Geiger is acknowledged for the synthesis of the cyanine dye. We thank Dr. Kappenberger and the Swiss SPM User Laboratory at Empa for support with the AFM measurements.

\section{References}

[1] T.H. James, The Theory of the Photographic Process, fourth ed., Macmillan, New York, 1977.

[2] U. Lawrentz, W. Grahn, K. Lukasznk, C. Klein, R. Wortmann, A. Feldner, D. Scherer, Chemistry - A European Journal 8 (7) (2002) 1573-1590.

[3] O.I. Tolmachev, N.V. Pilipchuk, O.D. Kachkovsky, Y.L. Slominski, V.Y. Gaywronsky, E.V. Shepelyavyy, S.V. Yakunin, M.S. Brodyn, Dyes and Pigments 74 (1) (2007) 195-201.

[4] M. Del Zoppo, A. Bianco, G. Zerbi, Synthetic Metals 124 (1) (2001) 183-184.

[5] H. Mustroph, M. Stollenwerk, V. Bressau, Angewandte Chemie - International Edition 45 (13) (2006) 2016-2035.
[6] J.H. Flanagan, S.H. Khan, S. Menchen, S.A. Soper, R.P. Hammer, Bioconjugate Chemistry 8 (5) (1997) 751-756.

[7] K. Licha, Contrast Agents for Optical Imaging. In: Contrast Agents II, vol. 222, 2002, pp. 1-29.

[8] R.O. Loutfy, J.H. Sharp, Photographic Science and Engineering 20 (4) (1976) 165-174.

[9] C.P. Chen, B.M. Zhou, D.H. Lu, G.G. Xu, Journal of Photographic Science 43 (4) (1995) 134-135.

[10] Y.S. Chen, Z.H. Zeng, C. Li, W.B. Wang, X.S. Wang, B.W. Zhang, New Journal of Chemistry 29 (6) (2005) 773-776.

[11] S.L. Li, K.J. Jiang, K.F. Shao, L.M. Yang, Chemical Communications (26) (2006) 2792-2794.

[12] K. Sayama, S. Tsukagoshi, T. Mori, K. Hara, Y. Ohga, A. Shinpou, Y. Abe, S. Suga, H. Arakawa, Solar Energy Materials and Solar Cells 80 (1) (2003) 47-71.

[13] Q.H. Yao, F.S. Meng, F.Y. Li, H. Tian, C.H. Huang, Journal of Materials Chemistry 13 (5) (2003) 10481053.

[14] M. Liang, W. Xu, F.S. Cai, P.Q. Chen, B. Peng, J. Chen, Z.M. Li, Journal of Physical Chemistry C 111 (11) (2007) $4465-4472$.

[15] J. Yang, C.C. Chen, H.W. Ji, W.H. Ma, J.C. Zhao, Journal of Physical Chemistry B 109 (46) (2005) 21900-21907.

[16] T.X. Wu, T. Lin, J.C. Zhao, H. Hidaka, N. Serpone, Environmental Science and Technology 33 (9) (1999) 13791387.

[17] F.A. Castro, A. Faes, T. Geiger, C.F.O. Graeff, M. Nagel, F. Nuesch, R. Hany, Synthetic Metals 156 (14-15) (2006) 973978.

[18] F.S. Meng, K.C. Chen, H. Tian, L. Zuppiroli, F. Nuesch, Applied Physics Letters 82 (21) (2003) 3788-3790.

[19] F. Nuesch, G. Tornare, L. Zuppiroli, F.S. Meng, K.C. Chen, H. Tian, Solar Energy Materials and Solar Cells 87 (1-4) (2005) 817-824.

[20] S. Cho, K. Lee, J. Yuen, G.M. Wang, D. Moses, A.J. Heeger, M. Surin, R. Lazzaroni, Journal of Applied Physics 100 (11) (2006) 114503.

[21] P. Peumans, S. Uchida, S.R. Forrest, Nature 425 (6954) (2003) 158-162.

[22] Y. Shao, S. Sista, C.W. Chu, D. Sievers, Y. Yang, Applied Physics Letters 90 (10) (2007) 103501.

[23] X.N. Yang, J. Loos, S.C. Veenstra, W.J.H. Verhees, M.M. Wienk, J.M. Kroon, M.A.J. Michels, R.A.J. Janssen, Nano Letters 5 (4) (2005) 579-583.

[24] A.J. Heeger, Angewandte Chemie - International Edition 40 (14) (2001) 2591-2611.

[25] B.A. Gregg, S.G. Chen, H.M. Branz, Applied Physics Letters 84 (10) (2004) 1707-1709.

[26] J.G. Xue, S.R. Forrest, Physical Review B 69 (24) (2004).

[27] A. Nollau, M. Pfeiffer, T. Fritz, K. Leo, Journal of Applied Physics 87 (9) (2000) 4340-4343.

[28] J. Drechsel, B. Mannig, F. Kozlowski, M. Pfeiffer, K. Leo, H. Hoppe, Applied Physics Letters 86 (24) (2005) 244102.

[29] G.A. Chamberlain, Journal of Applied Physics 53 (9) (1982) 6262-6269.

[30] A.C. Arias, M. Granstrom, D.S. Thomas, K. Petritsch, R.H. Friend, Physical Review B 60 (3) (1999) 1854-1860.

[31] K. Nakayama, Y. Matsui, M. Yokoyama, Japanese Journal of Applied Physics Part 1 - Regular Papers Brief Communications and Review Papers 44 (1B) (2005) 633-635.

[32] C.P. Kelly, C.J. Cramer, D.G. Truhlar, Journal of Physical Chemistry B 111 (2) (2007) 408-422. 
[33] T. Suzuki, Y. Maruyama, T. Akasaka, W. Ando, K. Kobayashi, S. Nagase, Journal of the American Chemical Society 116 (4) (1994) 1359-1363.

[34] N. Koch, A. Kahn, J. Ghijsen, J.J. Pireaux, J. Schwartz, R.L. Johnson, A. Elschner, Applied Physics Letters 82 (1) (2003) 70-72.

[35] T.D. Anthopoulos, T.S. Shafai, Applied Physics Letters 82 (10) (2003) 1628-1630.

[36] K. Kawano, R. Pacios, D. Poplavskyy, J. Nelson, D.D.C. Bradley, J.R. Durrant, Solar Energy Materials and Solar Cells 90 (20) (2006) 3520-3530.

[37] A. Geiser, B. Fan, H. Benmansour, F. Castro, J. Heier, B. Keller, K.E. Mayerhofer, F. Nüesch, R. Hany, Solar Energy Materials and Solar Cells (2007) accepted for publication.

[38] M. Rusu, J. Strotmann, M. Vogel, M.C. Lux-Steiner, K. Fostiropoulos, Applied Physics Letters 90 (15) (2007) 153511.
[39] P. Chen, J. Li, Z.G. Qian, D.S. Zheng, T. Okasaki, M. Hayami, Dyes and Pigments 37 (3) (1998) 213-222.

[40] G.W. Byers, S. Gross, P.M. Henrichs, Photochemistry and Photobiology 23 (1) (1976) 37-43.

[41] H. Horiuchi, S. Ishibashi, S. Tobita, M. Uchida, M. Sato, K. Toriba, K. Otaguro, H. Hiratsuka, Journal of Physical Chemistry B 107 (31) (2003) 7739-7746.

[42] P. Wardman, Journal of Physical and Chemical Reference Data 18 (4) (1989) 1637-1755.

[43] C.P. Chen, B.M. Zhou, D.H. Li, G.G. Xu, Journal of Photochemistry and Photobiology A - Chemistry 89 (1) (1995) 25-29.

[44] Y. Yamakoshi, N. Umezawa, A. Ryu, K. Arakane, N. Miyata, Y. Goda, T. Masumizu, T. Nagano, Journal of the American Chemical Society 125 (42) (2003) 1280312809 . 


\section{Organic Electronics}

Corrigendum

\section{Enhanced cyanine solar cell performance upon oxygen doping (Organic Electronics 9 (2008) 85-94)}

\section{Bin Fan ${ }^{\mathrm{a}}$, Roland Hany ${ }^{\mathrm{a}}$, Jacques-Edouard Moser ${ }^{\mathrm{b}}$, Frank Nüesch ${ }^{\mathrm{a}, *}$}

${ }^{a}$ Laboratory for Functional Polymers, Swiss Federal Laboratories for Materials Testing and Research, Empa, Überlandstrasse 129, CH-8600 Dübendorf, Switzerland

${ }^{\mathrm{b}}$ Institute of Chemical Sciences and Engineering, Ecole Polytechnique Fédérale de Lausanne, CH-1015 Lausanne, Switzerland

Error in Fig. 9

There is an error in the units on the vertical axis in Fig. 9. The correct units are $\mathrm{S} / \mathrm{m}$ :

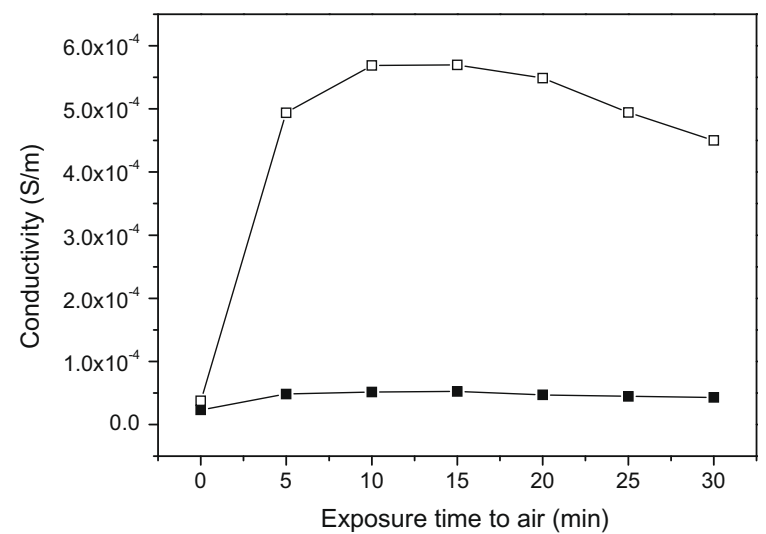

Fig. 9. Conductivity measurements of $\mathrm{Cy}-5$ films as a function of exposure time to ambient atmosphere in the dark (full squares) and under white light irradiation (empty squares). In both cases, conductivity measurements were performed in the absence of light.

E-mail address: frank.nueesch@empa.ch (F. Nüesch). 